\title{
Epidemiologic Disparities in Colon Cancer Screening and Adherence during the COVID-19 Pandemic: A Retrospective Cohort Analysis
}

\section{Eugene Chiemeka Nwankwo ( $\square$ eugene.nwankwo@health.slu.edu )}

Saint Louis University School of Medicine https://orcid.org/0000-0003-2031-8932

\section{Christian Hendrix}

Saint Louis University School of Medicine

\section{Kelvin Pollard}

Saint Louis University School of Medicine

\section{Chad Kallal}

Saint Louis University

\section{Tim Cruschiel}

Saint Louis University

\section{Frederick Buckhold III}

Saint Louis University Department of Internal Medicine

\section{Christine Y Hachem}

Saint Louis University Department of Internal Medicine, Division of Gastroenterology \& Hepatology

\section{Research Article}

Keywords: Colon cancer, screening, FIT, fecal testing, Equity, Diversity

Posted Date: January 11th, 2022

DOl: https://doi.org/10.21203/rs.3.rs-1191123/v1

License: (c) (i) This work is licensed under a Creative Commons Attribution 4.0 International License. Read Full License

Version of Record: A version of this preprint was published at International Journal of Colorectal Disease on March 11th, 2022. See the published version at https://doi.org/10.1007/s00384-022-04118-6. 


\section{Abstract}

Background: Colorectal cancer (CRC) is the second-leading cause of death in the US. Despite the National Colorectal Roundtable goal of achieving $80 \%$ adherence, CRC screening remains underutilized, especially in the underinsured populations. Screening programs have been heavily disrupted during the COVID-19 pandemic. Aim: This research was performed to explore the impact of the pandemic on the existing ethnic and gender disparities prevalent in CRC screening. Methods: Patients were identified 1 year before and after COVID-19 precautions began, using March 1, 2020, as the inflection point. For each year, the primary inclusion criterion was an ordered colonoscopy. The outcome of interest was a colonoscopy performed. Differences by year and race were assessed using Chi-square analysis. A cohort of 1413 patients between age 45 and 75 for whom a colonoscopy was ordered was selected from EHR at a single large institution. 897 patients were in the pre-COVID group, and 516 were in the post-COVID group. Results: There was a $51 \%$ reduction in screening colonoscopies performed. White patients had a decrease of $49 \%$, and African Americans had a 55\% reduction. Stool testing increased from $47 \%$ prior to the pandemic to $94 \%$ during the pandemic representing a greater than $100 \%$ increase in stool testing uptake. Conclusion: The true impact of COVID-19 on colorectal cancer is yet to be uncovered as future mortality estimates from CRC are ongoing. Due to the widespread closure of endoscopy centers and delay in screening, we believe that the pandemic worsened the screening disparities most prevalent among minority populations. Our retrospective analysis over the last two years points to the drastic reduction of screening for all races, and especially for African Americans. As life gradually returns to normal, it would be interesting to see how the past year has impacted the incidence and prevalence of CRC.

\section{Introduction}

Colorectal cancer (CRC) is the second leading cause of cancer-related deaths in the United States. ${ }^{1}$ There is clear evidence that screening with colonoscopy and stool-based tests is cost-effective. However, only $67 \%$ of adults between the ages of 50 and 75 were up to date with CRC screening in 2019. ${ }^{2}$ This number decreased to only $44 \%$ among individuals from uninsured and underserved populations in 2019.4,4 These metrics are significantly lower than the National Colorectal Roundtable goal of $80 \%$ adherence to CRC screening. ${ }^{5}$

Before the 2019 coronavirus disease (COVID-19) pandemic, there was a progressive increase in colorectal cancer screening across all races and insurance statuses from $67.4 \%$ in 2016 to $68.8 \%$ in $2018 .{ }^{7}$ Such success could largely be attributed to changes in CRC guidelines such as decreasing the age of at-risk individuals to 45 , and increased uptake of less invasive and less expensive screening modalities such as stool-based testing. ${ }^{7}$ During the pandemic low-income, underserved, and rural patient groups reported more challenges in their regular health care. The most cited barriers to care included finances and anxiety about contracting COVID-19. Moreover, patients on Medicare were more likely to face these challenges than those with private insurance. ${ }^{8}$ These challenges are likely to cause a significant reduction in screening and increased incidence of colorectal cancer for ethnic groups most at risk for CRC. 
The pandemic has also dramatically impacted numerous health and economic infrastructures. Given the rapid spread of the disease, various protective measures were employed. The Centers for Medicare \& Medicaid Services (CMS) recommended that all non-urgent surgical and medical procedures, including screening colonoscopy, be delayed. These measures decreased CRC screening in all individuals by $90 \%$, and CRC surgical procedures by $53 \%{ }^{6}$ This led to a subsequent decline in CRC diagnoses by $32 \% .{ }^{6}$

The objective of this single-center retrospective study was to quantify the effects of COVID-19 on screening rates across all races with more emphasis on those races most afflicted by colorectal cancer. We compared the rates of colonoscopy screening in a matched cohort of patients before and during the pandemic. Our findings contribute strong evidence that the circumstances surrounding the ongoing pandemic have further widened the disparities among medically underserved ethnic groups.

\section{Methods}

Patient data were collected from health system electronic health records (EMR) at Saint Louis University (SLU) Hospital, SLUCare Outpatient Endoscopy Center, and SLUCare Gastroenterology \& Hepatology Clinics. Patients were identified 1 year before (pre-COVID) and after COVID-19 precautions began (postCOVID), using March 1, 2020, as the inflection point. For each year, the primary inclusion criterion was an ordered colon cancer screening. A cohort of 1303 patients between age 45 and 75 for whom a colonoscopy was ordered was identified. Colonoscopy, FIT-DNA, FOBT, and CT colonography orders were identified using updated CPT and HCPCS codes recommended in the ASGE and Star Measure 2021 guidelines. Individuals who underwent colonoscopy or fecal testing as a diagnostic measure and those who already had a prior total colectomy were excluded (Supplemental Table 1).

Orders were cross matched with endoscopy encounters marked as completed or closed within the EMR, indicating that a particular patient was present and billed for a performed procedure. The race was determined by patient self-reported input into the EMR system. Individuals of Hispanic descent were indistinct from other races and were categorized as White in the EMR.

\section{Results}

\section{Demographics}

A total of 1413 individuals had colon cancer screening (including stool tests) ordered - 897 pre-COVID, and 516 post-COVID. 762 underwent scheduled screening - 388 pre-COVID, and 326 post-COVID. The mean ages were 61 and 59.8 in the pre-and post-COVID groups respectively $(p=0.0053)$. Among the individuals who were ordered cancer screening in the pre-covid cohort, 69.8\% $(n=626)$ were White, $27.3 \%$ $(n=244)$ African American, and 2.9\% $(n=26)$ were Asian (Table 2). In regard to performed colon cancer screening in the pre-COVID group, percent composition of the group was similar, including $66.9 \%(\mathrm{n}=$ 259) White, 29.8\% African American $(n=115), 2.6 \%$ Asian $(n=10)$, and $0.9 \%(n=3)$ American Indian. 
In the post-covid cohort, individuals who had CRC screening ordered comprised $68.8 \%(n=447), 28.9 \%$ ( $n$ $=187)$ African American, $2 \%(n=13)$ Asian or Pacific Islander, and $0.6 \%(n=3)$ were Native American or American Indian. Regarding performed colon cancer screening in the post-COVID group, there were $69.8 \%$ $(n=227)$ White, $29.8 \%$ African American $(n=88), 2.6 \%$ Asian $(n=9)$. There were no American Indian individuals in this cohort. Demographic characteristics of this population are further broken down in Figure 1.

\section{Overall Screening Rates Between Pre-Covid \& Post-Covid Groups}

During the pandemic, the total number of ordered screening colonoscopies was 481 compared to 822 a year prior, indicating a $41.5 \%$ decrease in scheduled colon cancer screening. On the other hand, there was a sizable increase in fecal testing (Cologuard and FIT) observed. There were 75 total kits ordered before the inflection point, and 163 ordered during the covid period - a $117 \%$ increase in provider utilization at our institution. While there was a $43 \%$ reduction in screening colonoscopies performed in the post-COVID period, fecal testing showed a screening rate of $94.4 \%$ (154/163) (Figure 2). CT colonography was the least ordered with only 6 orders placed pre-covid and none in the post-covid period.

\section{Ethnic \& Gender Differences in Colon Cancer Screening}

The overall cancer screening rate before COVID was 48.6\% (436/897) (Figure 2). Screening colonoscopies alone yielded a rate of $43 \%$ before the onset of COVID. The screening rate for women was $58 \%$, compared to $42 \%$ for men. White patients had a screening rate of $41 \%(236 / 567)$. African American patients had rates of $45.7 \%$ (105/230). Asian Americans were screened at a rate of $40.9 \%(9 / 22)$. Native Americans had the highest screen rate at 100\% (3/3) (Figure 3).

Furthermore, screening colonoscopy rates between both groups decreased from $43 \%$ (353/822) in the pre-COVID to $36 \%$ (172/481) in the post-COVID group. The reduction in colon cancer screens performed varied among ethnic groups during the pandemic. For White patients, the screening rate was $36.3 \%$ - a decrease of $49 \%$. African Americans had a post-covid screening rate of 33.8\% (47/139) representing a reduction of $55 \%$. Asian Americans had a screening rate of $62.5 \%(5 / 8)$ which was an increase of $52.8 \%$. There was no considerable difference in screening patterns based on gender.

Lastly, stool testing showed slightly different epidemiologic trends. There was an overall increase from $47 \%$ (35/75) to $94 \%$ (154/164) between the pre-and post-covid periods. By ethnicity, screening rates by stool testing for Whites before COVID were 38.9\% (23/59), 71.4\% (10/14) for African Americans, and 25\% (1/4) in Asians. In the Post-COVID period White patients had a screening rate of 92.2\% (107/116), African Americans had a rate of $87.5 \%$ (42/48), Asians and American Indians were 100\% each (Figure 1).

\section{Comparisons in Screening Between the First and Last half of the COVID-19 Pandemic}


During the pandemic, safety measures were implemented gradually. Our results were further broken down into 6-month periods during the pandemic Supplemental Table 2. There were no significant differences in screening rates during the first 6 months (March 2020 - August 2020) compared to the last 6 months (September 2020 - March 2021) of the pandemic.

\section{Discussion}

The true impact of COVID-19 on colorectal cancer is yet to be uncovered as true mortality estimates from CRC are still unknown. Due to the widespread closure of endoscopy centers and delay in screening, we believe that the pandemic worsened the screening disparities most prevalent among minority populations. To the best of our knowledge, this is the first study to assess the real-time impact of the COVID-19 pandemic on the screening and management of colorectal cancer in the United States. Our retrospective analysis of CRC screening over the past two years points to a drastic reduction in screening for all races, and especially for those populations most medically underserved. Regarding health services, "underserved" refers to those individuals or populations who are disadvantaged because of ability to pay, ability to access care, ability to access comprehensive healthcare, or other disparities for reasons of race, religion, language group, or social status.

This study further elaborates that screening colonoscopies decreased during the COVID pandemic. Such reduction was evident across all races; and most in African American patients. On the other hand, the pandemic may have improved the uptake of other screening methods including stool tests. The observed differences in screening rates could be ascribed to several behaviors and policies implemented during the pandemic. Our data indicated a $41.5 \%$ decrease in colonoscopy screening orders, and over $100 \%$ increase in non-invasive testing during the post-covid period compared to pre-covid. Overall reduction in ordered CRC screening was likely a reflection of the mandate to suspend nonurgent medical procedures and surgeries and a likely reduction in overall clinic visits. This recommendation directly led to the suspension of colonoscopies for CRC screening and surveillance. On the other hand, uptake of other screening modalities such as the FIT was inadequate to fill the void left by the reduction of colonoscopies. ${ }^{4}$ Although there was a slight increase in the orders placed for non-invasive CRC screening (FIT, Cologuard, and CT Colonography) and an even greater uptake by patients, these positive behaviors were not enough to offset the void left by such drastic reductions on colonoscopy screens.

In addition to the overall screening rates, we also observed reductions in colon cancer screening performed across all races during the pandemic with the largest decrease being in African Americans. Historically, CRC disproportionately affects members of minority groups. African Americans and Native Americans have the highest incidence of CRC; African Americans have the highest mortality, and Hispanics have the lowest CRC screening rates. ${ }^{1}$ These discrepancies have been ascribed to various factors, including psychosocial and economic barriers to health care and screening. The documented barriers include fear of anesthesia or a terminal diagnosis, financial difficulties or lack of assistance, logistical challenges, and lower priority. ${ }^{9}$ The pause in elective procedures and the economic impact of the pandemic may have exacerbated these existing barriers to care, especially for those patients 
inhabiting rural locations in Missouri and Illinois. Limitations of our study include its nature as a chart review, the self-reported nature of race which likely led to the inconsistent categorization of races, especially within the Hispanic cohort. Lastly, there were some coding limitations in our data collection. Some procedures particularly fecal testing (FIT or Cologuard) may have been coded as the same given that their orders are similar.

In its wake, the COVID pandemic likely delayed the diagnosis and worsened mortality of CRC across various groups, especially those already underserved in healthcare. Most of all, it is likely to have slowed the progress made in recent years to increase CRC screening rates and bridge the gap in health disparities.

\section{References}

1. Colorectal Cancer Screening Test Use* (\%), Adults 50 Years and Older by State, 2018.

2. Khalili, F. et al. Cost-Effectiveness Analysis of Colorectal Cancer Screening: A Systematic Review. (2020) doi:10.2147/RMHP.S262171.

3. Perisetti, A. et al. Colorectal cancer screening use among insured adults: Is out-of-pocket cost a barrier to routine screening? World J. Gastrointest. Pharmacol. Ther. 9, 31 (2018).

4. Balzora, S. et al. Impact of COVID-19 on colorectal cancer disparities and the way forward. Gastrointest. Endosc. 92, 946 (2020).

5. May, F. P., Yang, L., Corona, E., Glenn, B. A. \& Bastani, R. Disparities in Colorectal Cancer Screening in the United States Before and After Implementation of the Affordable Care Act. Clin. Gastroenterol. Hepatol. 18, 1796-1804.e2 (2020).

6. New Colorectal Cancer Diagnoses Fall by One-Third and Colonoscopies Grind to a Halt During Height of COVID-19. https://www.komodohealth.com/insights/2020/05/new-colorectal-cancer-diagnosesfall-by-one-third-and-colonoscopies-grind-to-a-halt-during-height-of-covid-19.

7. Colorectal Cancer Statistics | CDC. https://www.cdc.gov/cancer/colorectal/statistics/index.htm.

8. Review of Barriers to Colorectal Cancer Screening in Hard-to-Reach Populations - Cancer Therapy Advisor. https://www.cancertherapyadvisor.com/home/cancer-topics/gastrointestinalcancers/colorectal-cancer-covid19-pandemic-barriers-treatment-screening/.

9. Muthukrishnan, M., Arnold, L. D. \& James, A. S. Patients' self-reported barriers to colon cancer screening in federally qualified health center settings. Prev. Med. Reports 15, 100896 (2019).

\section{Declarations}

All authors contributed to the study's conception and design. Material preparation, data collection, and analysis were performed by Eugene C Nwankwo, Jr., Chad Kallal, and Tim Cruschiel. The first draft of the manuscript was written by Eugene $\mathrm{C}$ Nwankwo, Jr and all authors commented on previous versions of the manuscript. All authors read and approved the final manuscript. 
Competing interests: The authors declare no competing interests.

Ethics statement: This is an observational study. The Saint Louis University Research Ethics and IRB Committee have confirmed that no ethical approval is required.

\section{Figures}

\section{Figure 1}

Summary of patients with ordered and performed colon cancer screening (colonoscopy and stool tests).

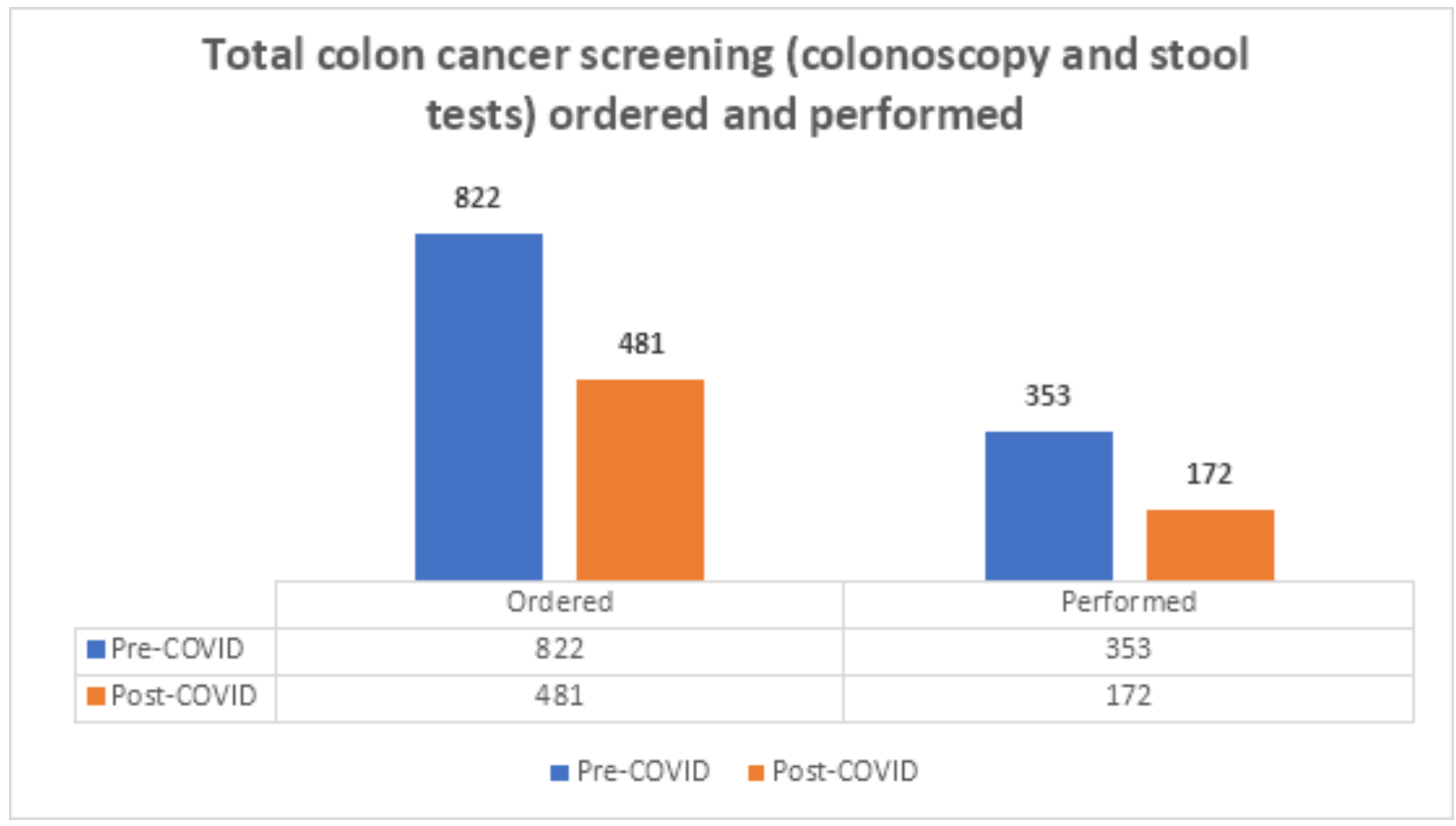

Figure 2

Comparison of total colon cancer screenings (colonoscopy and stool tests) ordered and performed in the pre-COVID and post-COVID periods. 


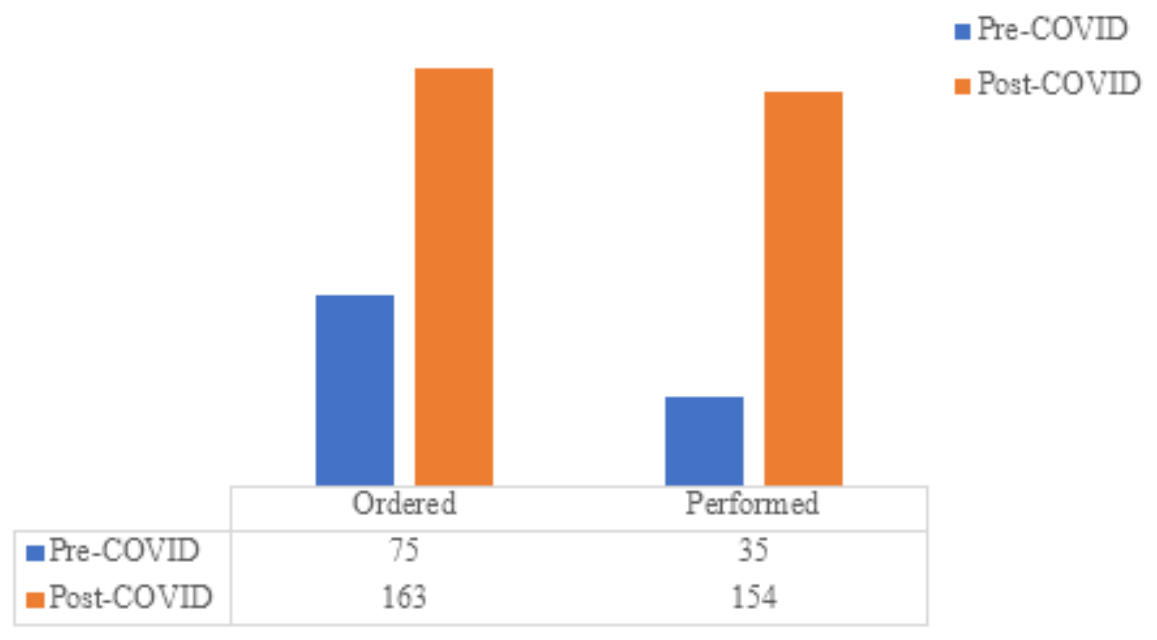

Figure 3

Adherence to non-invasive colon cancer screening increased three-fold during the pandemic. Screening rates were $94 \%$ in the post-COVID compared to $46 \%$ in the pre-COVID period.

\section{Figure 4}

A: Number of colonoscopies ordered for routine colon cancer screening reduced from the pre-COVID to post-COVID period. B: Colonoscopies performed decreased across all races. Screening in White patients decreased by $49 \%, 55 \%$ in African American, and increase of $52.8 \%$ in Asian American patients.

\section{Supplementary Files}

This is a list of supplementary files associated with this preprint. Click to download.

- SupplementalTablesCRCCOVID.docx 